Abanico Agroforestal. Enero-Diciembre 2021; 3:1-13. http://dx.doi.org/10.37114/abaagrof/2021.2 Artículo Original. Recibido: 18/12/2020. Aceptado: 20/02/2021. Publicado: 19/03/2021. Clave: e2020-16.

\title{
Análisis de la estructura del cuerno del toro de lidia mediante análisis de imagen. Efecto del enfundado
}

\author{
Analysis of the structure of the lidia bull horn through image analysis. Horn sheath \\ effect
}

\section{Lomillos-Pérez Juan Manuel ${ }^{1 *}$ ID, José Manuel Gonzalo² ID, Marta Elena Alonso ${ }^{31 D}$}

1Departamento de Producción y Sanidad Animal, Salud Pública Veterinaria y Ciencia y Tecnología de los Alimentos. Facultad de Veterinaria. Universidad Cardenal Herrera-CEU. C/ Tirant lo Blanc, 7. 46115 Alfara del Patriarca - Valencia. España. ${ }^{2}$ Departamento de Medicina, Cirugía y Anatomía Veterinaria. Facultad de Veterinaria de León. Universidad de León. Campus de Vegazana s/n. 24071. León. España. ${ }^{3}$ Departamento de Producción Animal. Facultad de Veterinaria de León. Universidad de León. Campus de Vegazana s/n. 24071. León. España. *Autor de correspondencia. juan.lomillos@uchceu.es,jm.gonzalo.orden@unileon.es, marta.alonso@unileon.es

\section{RESUMEN}

La cornamenta del toro de Lidia es la parte anatómica más importante del animal, ya que le confiere su carácter ofensivo y a la vez le dota de pureza e integridad. En la actualidad, en muchas de las explotaciones se protege el cuerno durante el último año de cría del toro con una funda de fibra de vidrio. Con el presente trabajo se pretende estudiar la estructura interna del cuerno del toro de Lidia, analizando la posible influencia del enfundado en la misma. Para ello se han recogido cuernos de 55 toros (4-5 años), contando con un grupo de individuos enfundados de 25 animales. Se ha recogido información biométrica del cuerno y de la edad del animal. Un cuerno de cada animal ha sido sometido a estudio de imagen mediante radiografía, densitometría y resonancia magnética. El estudio radiológico ofreció gran información sobre la estructura interna del cuerno, siendo un buen método para la valoración de la integridad del mismo. La cornamenta de los animales no enfundados presentó mayores valores de área ósea, contenido y densidad mineral a nivel de la zona ósea distal, lo que revela un efecto nocivo del enfundado. Este podría derivar en una mayor predisposición a una eventual fractura, sin embargo, no se observaron diferencias entre grupos en el estudio realizado mediante resonancia magnética.

Palabras clave: toro de Lidia, raza de Lidia, cuernos.

\begin{abstract}
The Lidia bull honrs are the most important anatomical part of the animal, since it gives it its offensive character and at the same time gives it purity and integrity. Today, in many of the farms, the horn is protected during the last year of breeding with a fiberglass cover. This paper aims to study the internal structure of the fighting bull's horn, analyzing the possible influence of the sheath on it. For this, horns have been collected from 55 bulls (4-5 years), with a group of sheathed individuals of 25 animals. Biometric information has been collected on the horn and the age of the animal. One horn from each animal has undergone an imaging study using radiography, densitometry and magnetic resonance imaging. The radiological study offered great information on the internal structure of the horn, being a good method for evaluating the integrity of the horn. The antlers of the non-sheathed animals presented higher values of bone area, mineral content and mineral density at the level of the distal bone area, which reveals a harmful effect of the sheath. This could lead to a greater predisposition to a eventual fracture, however, no differences between groups were observed in the study performed by magnetic resonance imaging.
\end{abstract}

Keywords: Lidia bull, Lidia cattle, horns. 


\section{INTRODUCCIÓN}

Los cuernos del toro, son sin lugar a duda, un elemento trascendental en la fiesta taurina y en el rito del espectáculo; son el ícono de integridad y pureza del espectáculo mismo; sobre esta integridad se ha debatido y debate desde los inicios del toreo. La aproximación veterinaria hacia este aspecto debe realizarse desde un punto de vista técnico y profesional, y por tanto como una estructura anatómica del animal susceptible de sufrir diversas lesiones y patologías (Sotillo et al., 1996). Por ello, es fundamental el conocimiento de sus características anatómicas, histológicas, fisiológicas y patológicas que permitan diagnosticar y explicar las diferentes enfermedades o fraudes que le afecten (Alonso et al., 2016).

La literatura existente sobre los cuernos de las reses de lidia no es muy abundante. Los libros clásicos de exterior (Aparicio-Sánchez, 1960; Sañudo, 2009), tratan el tema de las encornaduras como un fanero más, pero no se refieren de forma específica a la res de lidia. Otros autores (Cossío, 1967; Barga, 1972) se refieren concretamente a dicha raza, pero hacen, más bien, una clasificación según su conformación.

A partir del descubrimiento del fraude del "afeitado" la literatura sobre conformación de los cuernos y su relación con la capacidad combativa de las reses ha sido más abundante (Trillo, 1961; Maubon, 1956; Llorente, 1980; Bobed, 1982; Martín, 1984; Fuente et al., 1999; Ezpeleta, 1999; Aparicio et al., 2000).

El cuerno es una producción epidérmica que se sitúa a ambos lados del testuz, sustentado en el saliente óseo del hueso frontal, adoptando forma cónica y alargada; se divide en 3 partes (figura 1): parte proximal o "cepa" (también llamada mazorca), parte media o "pala" y parte distal o "pitón" (Fernández, 2009). Como tal producto óseo, aparecen en su composición minerales como el calcio, hierro, magnesio, sodio, potasio y fósforo; dependiendo su dureza en gran medida, (Cabanas et al., 1994).

La encornadura del toro sufre un riesgo de deterioro, fundamentalmente en el último año de vida, a consecuencia de potenciales peleas, roces, contactos o golpes con el suelo, árboles, el vallado, comederos o las paredes de las mangas o corrales de manejo (Aparicio et al., 2000). Por ello, en los últimos años se ha popularizado el uso de un vendaje de fibra de vidrio para recubrir los cuernos de forma que se protejan hasta su lidia en la plaza (Lomillos et al. 2013). Es un material fácil de manejar, poroso y que se endurece rápidamente por polimerización con el agua, proporcionando buena consistencia (figura 2). La técnica consiste en inmovilizar al animal en el mueco y envolver el cuerno con este vendaje para protegerlo de cualquier agresión o roce. La parte distal del cuerno, es decir el pitón, se refuerza en muchos casos con un material más duro, tubos metálicos o similares, con el fin de disminuir el desgaste de la zona apical (Pizarro et al., 2008a y b). 


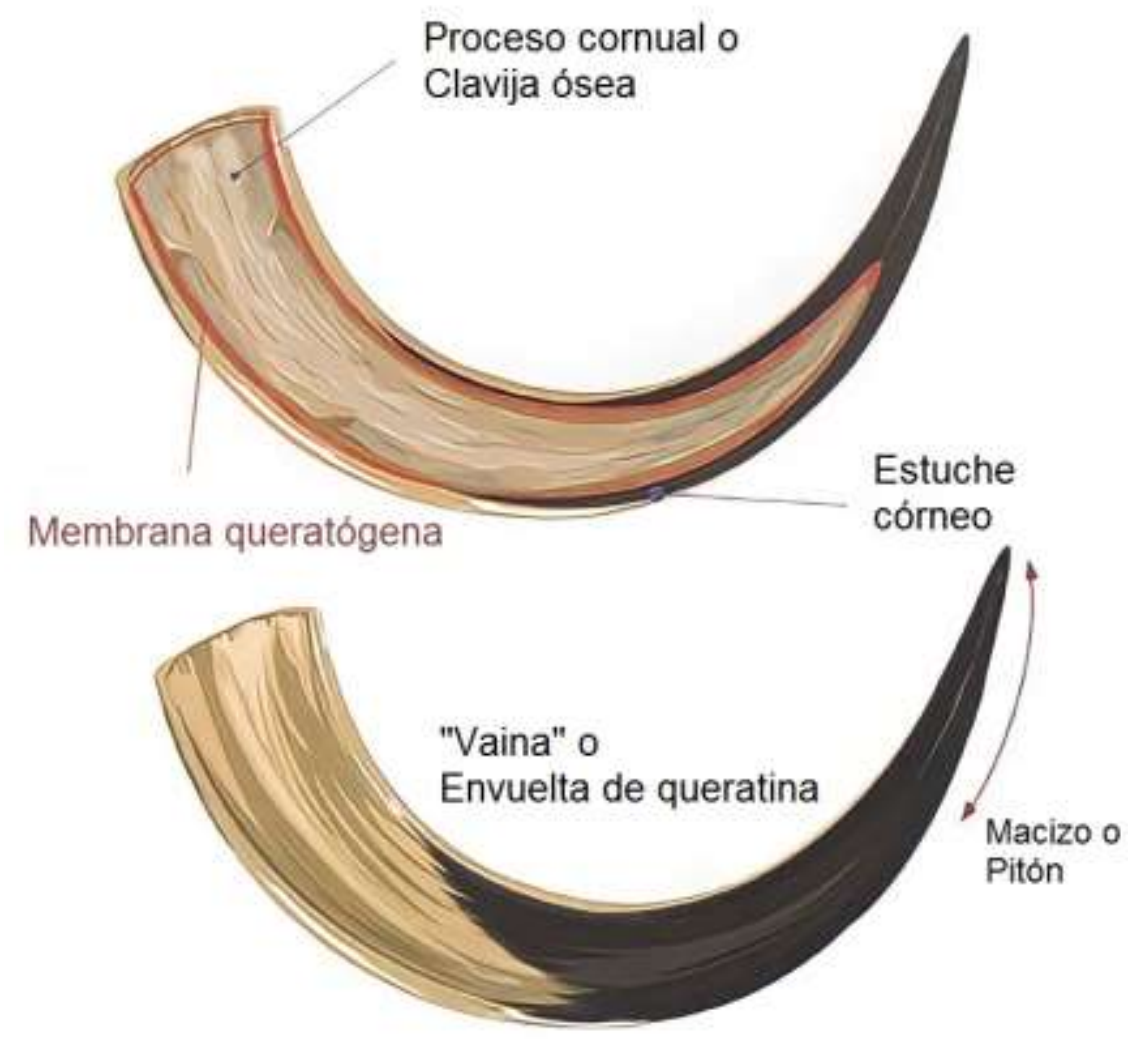

Figura 1. Anatomía del cuerno del toro (Calvo, 2005)

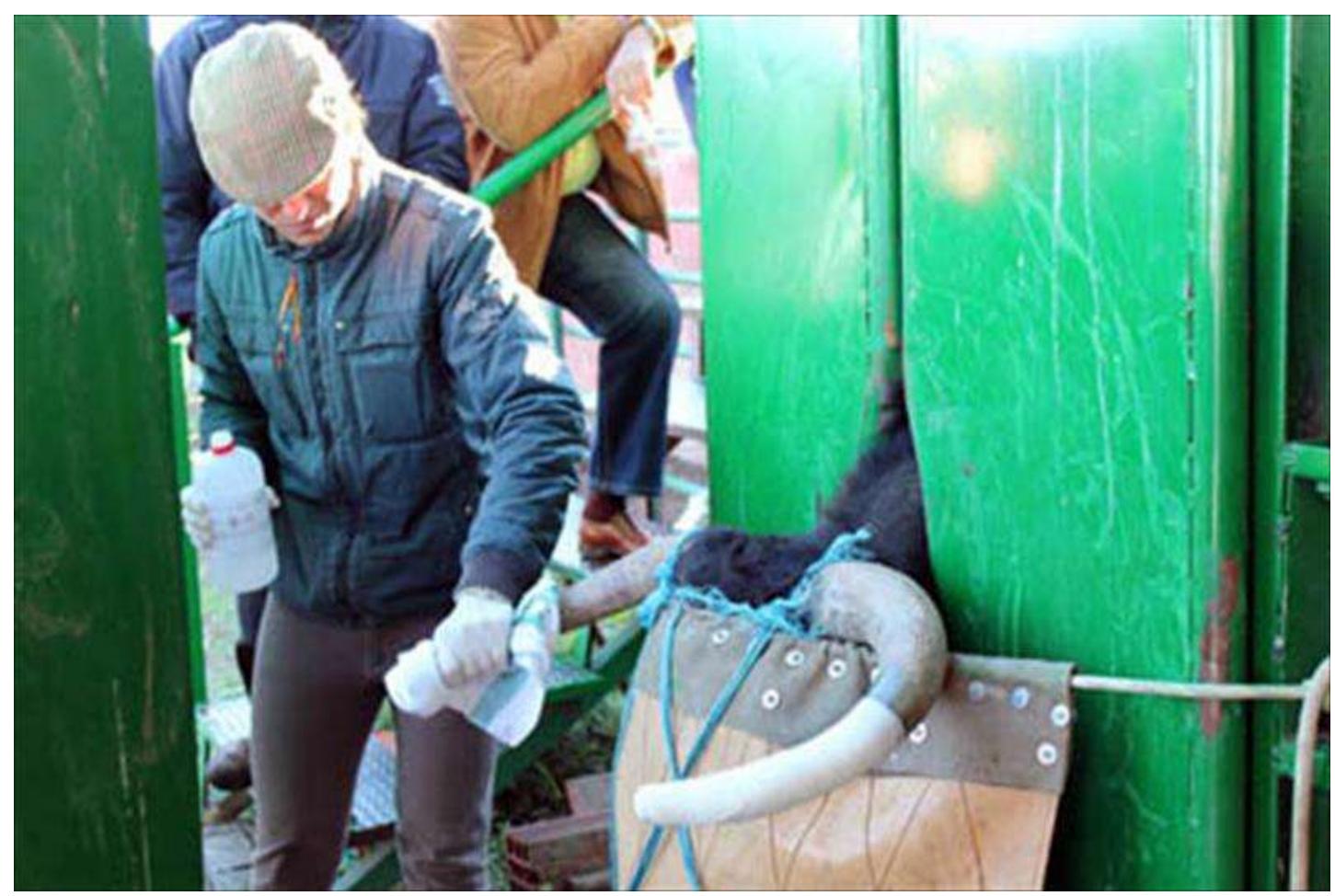

Figura 2. Manejo de enfundado (Fotografía: Julio Cesar Sánchez, 2018). 
Existe un debate sobre el uso legítimo de las fundas ante la evidente manipulación de los cuernos en los momentos de la colocación y retirada del vendaje. Sus defensores argumentan que esta práctica tiene el objetivo de conservar la integridad de los pitones; mientras que la legislación vigente habla de que el ganadero debe asegurar la "intangibilidad de las defensas del toro" (REAL DECRETO 176/1992).

Igualmente se desconoce cómo podría afectar este tipo de manipulación del cuerno a su estructura y, por ende, así predispondría la posibilidad de una fractura; puesto que algunos autores han observado una necrosis de la dermis cornual, con pérdida de estructura ósea y aparición de espacios huecos en el interior del cuerno, que podrían reducir su resistencia (Gómez et al., 2009).

Se han llevado a cabo algunos pequeños estudios de aproximación al efecto del enfundado, la dureza superficial que reflejan conclusiones dispares (Pizarro et al., 2008 a,b y 2009; Salamanca, 2009; Horcajada et al., 2009), pero no existen estudios sobre el efecto en la estructura interna de este órgano.

El objetivo de este trabajo es ampliar el conocimiento sobre la estructura interna de la cornamenta del toro de Lidia y arrojar luz sobre el posible efecto del enfundado sobre la misma.

\section{MATERIAL Y MÉTODOS}

Se han analizado la cornamenta de 55 toros de Lidia de 3 a 5 años, 25 de ellos que han sido enfundados 6 meses antes de su sacrificio.

Inicialmente se llevaron a cabo varias medidas del cuerno: longitud del cuerno (media de la longitud externa e interna) y diámetros vertical y horizontal en la cepa, pala y pitón mediante cinta métrica y calibre.

\section{Radiología}

Se procedió a realizar un análisis de imagen de las estructuras cornuales, utilizando un equipo de radiología de la marca Sedecal, recogiendo las imágenes en un chasis digital (CR) de la marca Fujifilm. Se realizaron radiografías inicialmente en 12 animales en ambos cuernos observándose una simetría manifiesta entre los dos cuernos en la parte ósea, por lo cual se decidió realizar las siguientes determinaciones, sólo en los cuernos derechos.

\section{Densitometría}

Dado que uno de los aspectos determinantes de la resistencia mecánica del cuerno es el grado de calcificación de las estructuras óseas que sirven de soporte al mismo, consideramos necesario realizar una evaluación de la densidad ósea de los procesos cornuales del hueso frontal, de manera que sea posible comprobar la existencia o no de influencia del enfundado a este nivel. Se utilizó un equipo de densitometría Hologic QDR-1000/W; efectuándose mediciones tanto de la mitad proximal del cuerno (base) como de la mitad distal (punta). Para determinar las dos regiones de estudio se utilizó como referencia el punto medio del cuerno localizado mediante laser (Figura 3). 


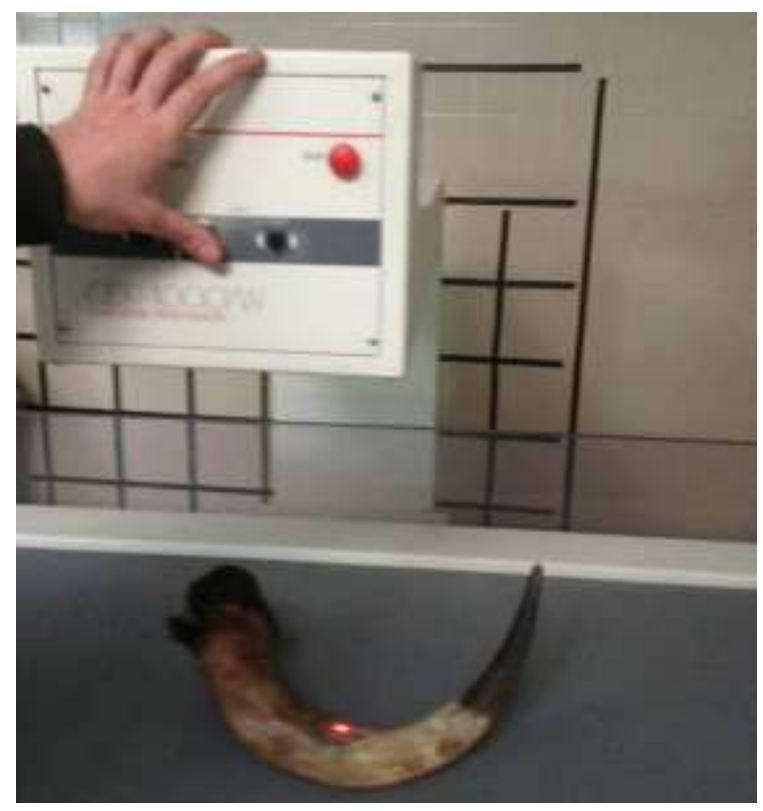

Figura 3. Localización del punto medio del cuerno mediante laser

A partir de ese momento se realizan una serie de haces radiológicos que permiten obtener valores de Contenido Mineral Óseo (BMC gr), y área ósea estudiada (cm2), a partir de estos cuales el programa informático del densitómetro calcula la Densidad Mineral Ósea (BMD gr/cm2).

\section{Resonancia Magnética}

Para este estudio se empleó un equipo de resonancia magnética cerrado de 3 Teslas, con imán superconductivo, marca General Electric Medical System; después de dos pruebas preliminares con una antena para muñeca y otra de cabeza se optó por realizar los estudios con una antena Cardio-array (figura 4), de la misma marca comercial. Las imágenes fueron procesadas mediante el programa de OsiriX, que permite realizar mediciones precisas sobre las mismas.

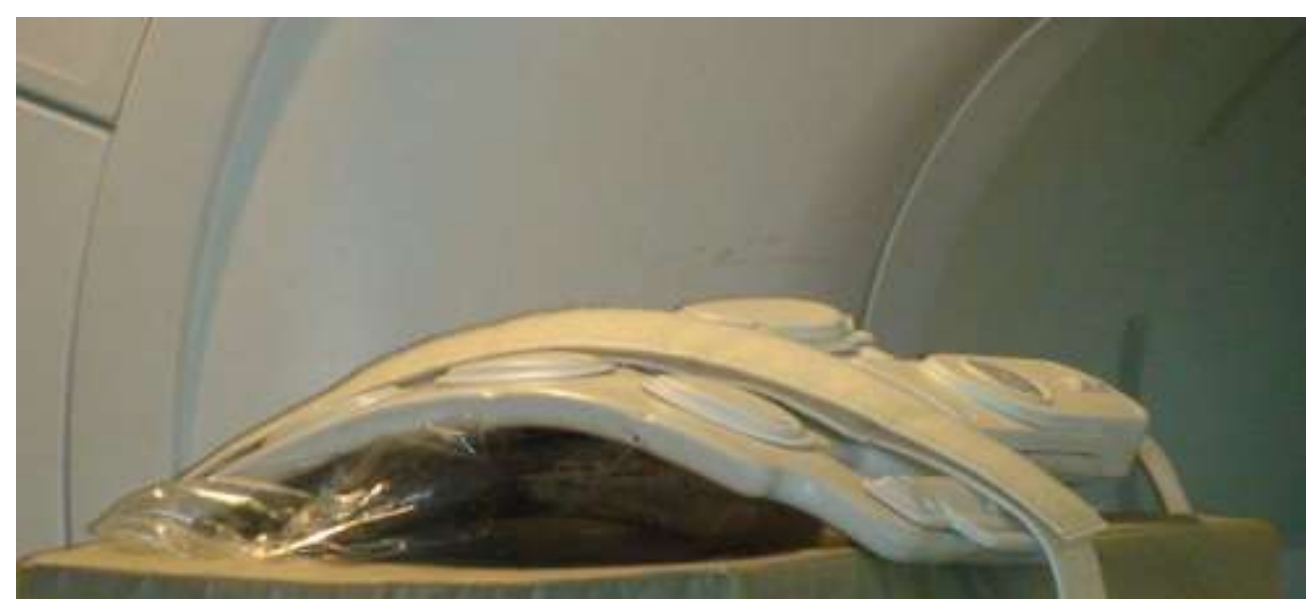

Figura 4. Cuerno cubierto con antena Cardio-array 
Los cuernos se colocaron sobre dos almohadillas para centrarlos en el imán, en una posición que correspondería a decúbito supino del animal; pero algo más rotados para aprovechar la zona de mayor intensidad magnética.

La técnica de estudio consistió en un localizador en 3 planos, con un segundo localizador también en tres planos complementario; sobre éstos se realizaron cortes coronales oblicuos, buscando el plano que abarcase una mayor longitud del cuerno y cubriendo con el resto de los cortes toda la anatomía (figura 5). Estos cortes eran de $5 \mathrm{~mm}$ de espesor separados entre ellos $0.5 \mathrm{~mm}$, lo que se traduce en una distancia de $5.5 \mathrm{~mm}$ entre cortes. Estas imágenes fueron adquiridas en T2 FRFSE y en T1SE.

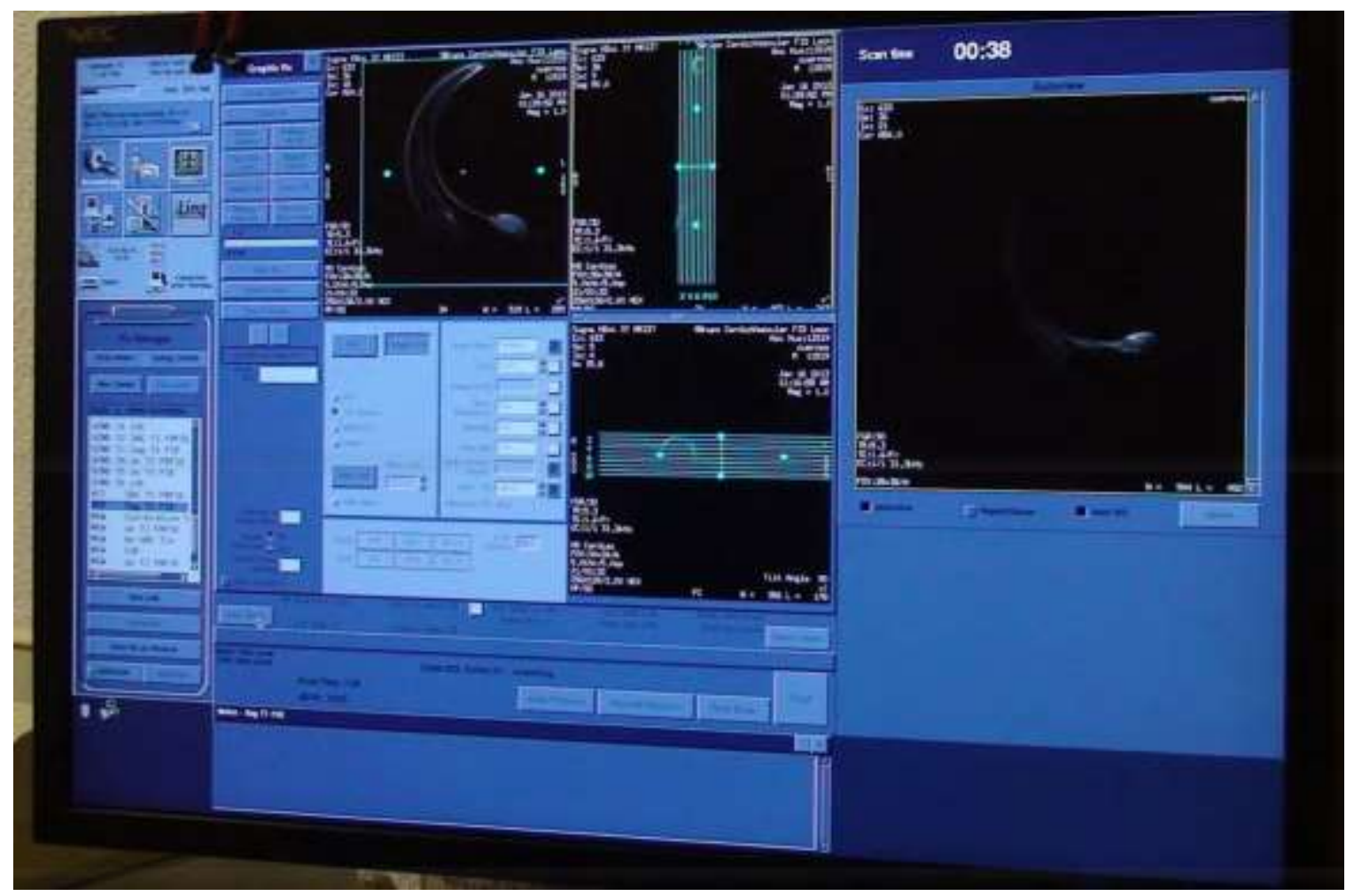

Figura 5. Imagen de los cortes coronales oblicuos de $5 \mathrm{~mm}$ de espesor

Sobre las anteriores imágenes se programaron los cortes axiales, perpendiculares a los anteriores con $6 \mathrm{~mm}$ de espesor y $14 \mathrm{~mm}$ entre cortes (figura 6); lo que nos da una distancia entre cortes de $20 \mathrm{~mm}$, que nos facilitara la comparación de resultados con el resto de las técnicas. Estas imágenes también fueron obtenidas en T2FRFSE y T1SE. 


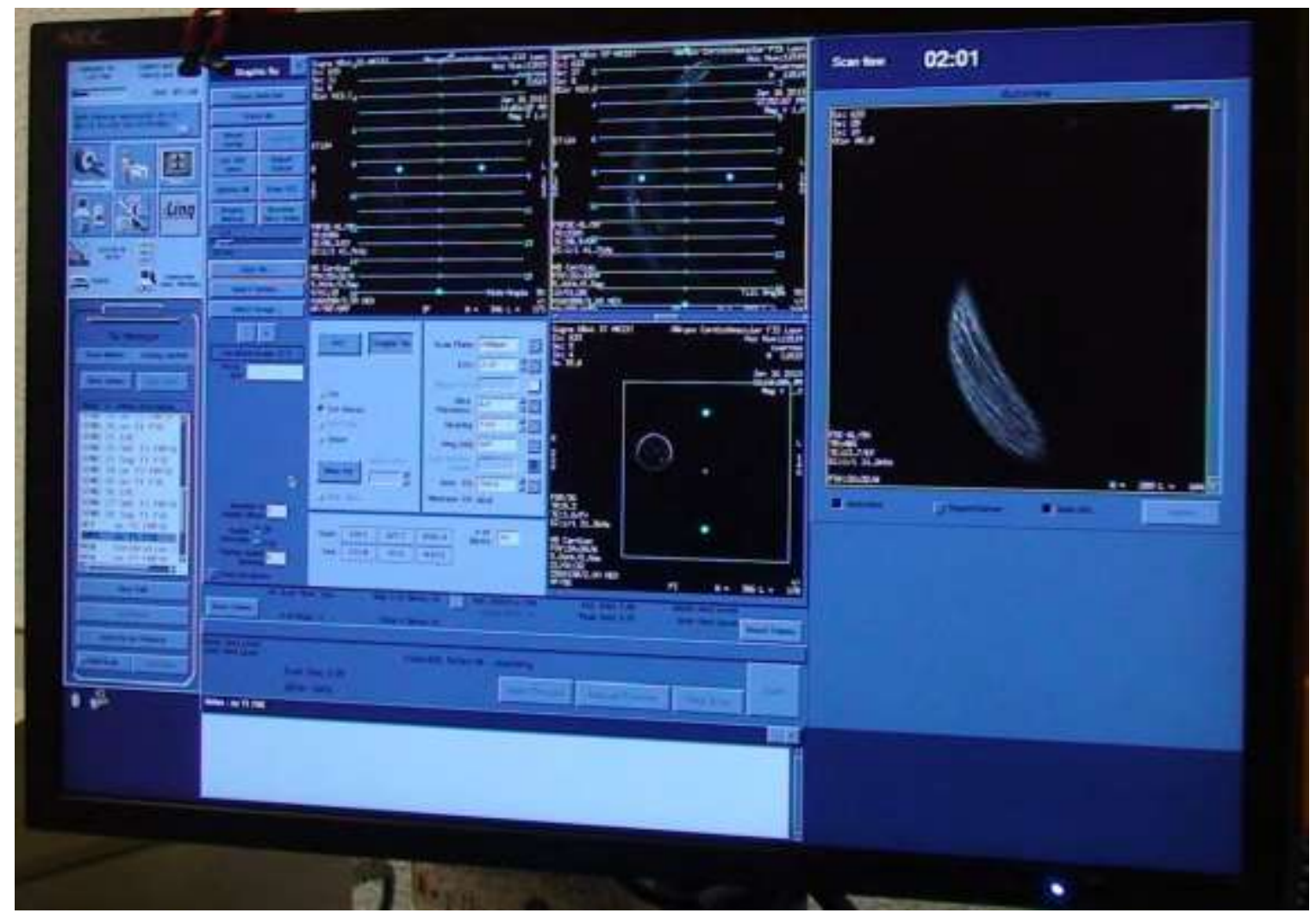

Figura 6. Imagen de los cortes axiales perpendiculares a los coronales de $6 \mathrm{~mm}$ de espesor

La orientación de estas series axiales hace que, al ser los cortes paralelos entre sí, los dos primeros y los dos últimos sean muy oblicuos, dándonos una imagen elíptica de la sección del hueso, siendo el resto de los cortes razonablemente perpendiculares al eje del cuerno.

Todos los datos fueron procesados mediante el programa SPSS, realizando un análisis de varianza (ANOVA) de una vía, considerando grupo 0 los animales no enfundados y grupo 1 los enfundados.

\section{RESULTADOS Y DISCUSIÓN}

\section{Radiología}

Mediante radiografía es posible distinguir perfectamente las diferentes partes del cuerno y por ende su estructura interna. No se encontraron alteraciones graves de la estructura interna de los cuernos, ni compatibles con procesos patológicos crónicos en la zona ósea de ninguno de los cuernos estudiados. Si bien se apreciaron algunas alteraciones en la zona del macizo como roturas o astillados, como puede verse en la parte superior izquierda de la figura 7, pero tanto en animales del grupo enfundado como no enfundado. 


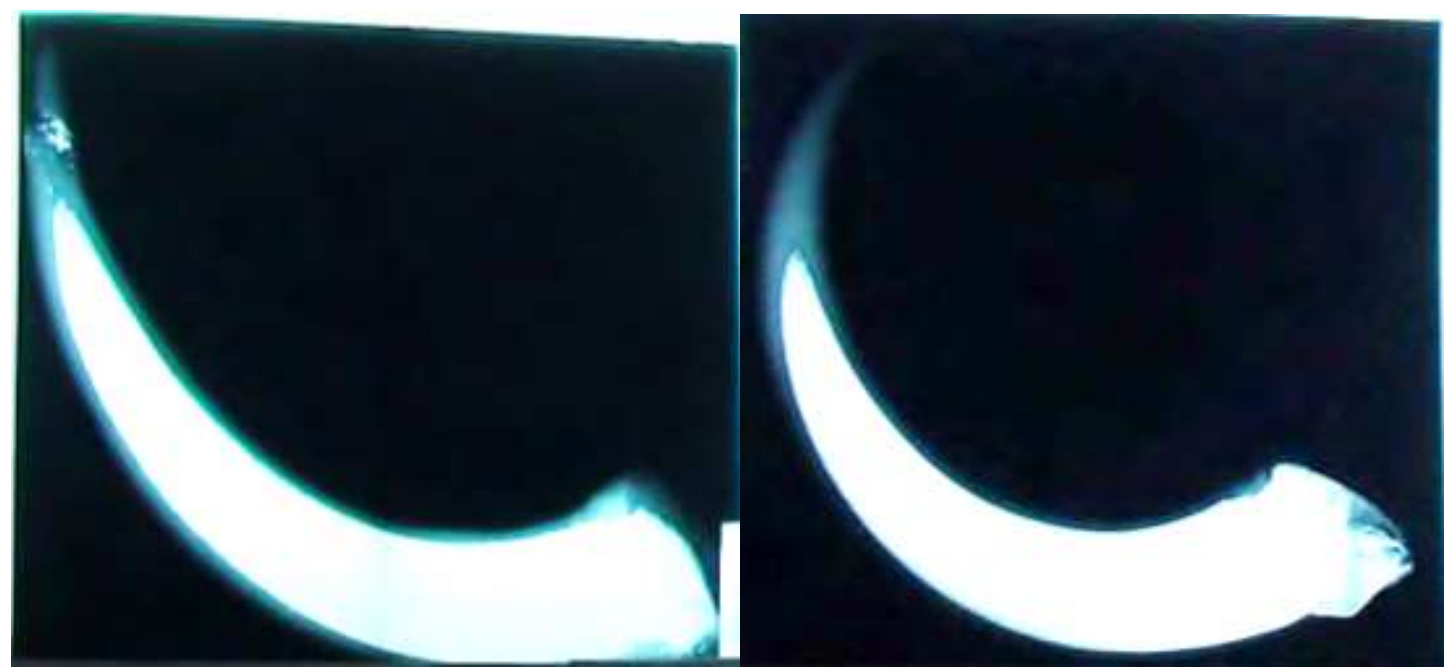

Figura 7. Imágenes radiográficas de un cuerno fracturado y uno sano

\section{Densitometría}

EI ANOVA de una vía realizado entre grupos en función de esta variable, dio diferencias significativas entre grupos en los valores referidos a la mitad distal del cuerno denominada punta, como puede verse en la tabla 1.

Tabla 1. ANOVA de los valores densitométricos obtenidos

\begin{tabular}{|c|c|c|c|c|c|c|c|c|}
\hline $\begin{array}{l}\text { Media } \\
\text { Gr } 0\end{array}$ & $\begin{array}{l}\text { Media } \\
\text { Gr } 1\end{array}$ & $\begin{array}{c}\text { Des. Est. } \\
0\end{array}$ & $\begin{array}{c}\text { Des. Est. } \\
1\end{array}$ & df & $d f$ & MS & $\mathrm{F}$ & $p$ \\
\hline 67.677 & 56.964 & 11.648 & 11.613 & 1 & 47 & 135.311 & 10.282 & 0.002 \\
\hline 120.931 & 97.057 & 26.256 & 24.867 & 1 & 47 & 657.656 & 10.507 & 0.002 \\
\hline 1.777 & 1.687 & 0.170 & 0.121 & 1 & 47 & 0.023 & 4.358 & 0.042 \\
\hline 129.756 & 123.395 & 18.680 & 20.360 & 1 & 47 & 378.255 & 1.297 & 0.261 \\
\hline 300.275 & 293.270 & 54.570 & 51.385 & 1 & 47 & 2827.126 & 0.210 & 0.649 \\
\hline 2.309 & 2.378 & 0.193 & 0.162 & 1 & 47 & 0.032 & 1.777 & 0.189 \\
\hline 421.207 & 390.326 & 70.647 & 70.330 & 1 & 47 & 4971.029 & 2.325 & 0.134 \\
\hline 197.433 & 180.359 & 25.750 & 29.710 & 1 & 47 & 761.176 & 4.643 & 0.036 \\
\hline
\end{tabular}

La zona proximal del cuerno no presentó diferencias entre los dos grupos de estudio, realizándose seguidamente una matriz de correlación entre los valores obtenidos por densitometría y las variables biométricas externas, cuyo resultado aparece en la tabla 2. 
Tabla 2. Matriz de correlación lineal entre las variables estudiadas por técnicas biométricas convencionales y densitometría

\begin{tabular}{ccccccccc} 
& $\begin{array}{c}\text { Long } \\
\text { Externa }\end{array}$ & $\begin{array}{c}\text { Long } \\
\text { Interna }\end{array}$ & $\begin{array}{c}\text { D Vert } \\
\text { Cepa }\end{array}$ & $\begin{array}{c}\text { D Hor } \\
\text { Cepa }\end{array}$ & $\begin{array}{c}\text { D Ver } \\
\text { Pala }\end{array}$ & $\begin{array}{c}\text { D Hor } \\
\text { Pala }\end{array}$ & $\begin{array}{c}\text { D Vert } \\
\text { Pitón }\end{array}$ & $\begin{array}{l}\text { D Hor } \\
\text { Pitón }\end{array}$ \\
\hline Área Punta & 0.155 & -0.007 & 0.350 & 0.482 & 0.301 & 0.581 & -0.464 & -0.176 \\
BMC Punta & 0.098 & -0.072 & 0.382 & 0.546 & 0.312 & 0.596 & -0.482 & -0.142 \\
BMD Punta & 0.047 & -0.112 & 0.368 & 0.465 & 0.259 & 0.466 & -0.428 & -0.006 \\
Área Base & 0.695 & 0.546 & 0.719 & 0.431 & 0.635 & 0.569 & -0.582 & -0.326 \\
BMC Base & 0.711 & 0.546 & 0.581 & 0.328 & 0.555 & 0.445 & -0.513 & -0.192 \\
BMD Base & 0.226 & 0.131 & -0.124 & -0.106 & -0.020 & -0.124 & -0.030 & 0.233 \\
BMC Total & 0.587 & 0.399 & 0.586 & 0.448 & 0.541 & 0.556 & -0.568 & -0.199 \\
Área Total & 0.596 & 0.422 & 0.685 & 0.508 & 0.603 & 0.651 & -0.620 & -0.317 \\
\hline
\end{tabular}

Las correlaciones marcadas son significativas a nivel de $p<0.05$

Cabe destacar, como no existe correlación del área, contenido mineral o densidad mineral de la punta con la longitud total del cuerno ni externa ni interna, pero sí con los diámetros horizontales de la cepa y pala, siendo inversa con el pitón. En una primera aproximación podemos interpretarlos como que los cuernos no enfundados tienen una mayor densidad, debido en cierta medida a su mayor grosor. Existe sin embargo otra posible explicación que radicaría en que la ausencia de protección de la funda hace que el hueso responda con una mayor calcificación ante los traumatismos, producidos de modo continuo en peleas, golpes y demás efectos mecánicos continuos; aspecto este último comprobado en humana.

\section{Resonancia Magnética}

Por último, a través de las resonancias magnética realizadas hemos observado con claridad los patrones de los canales vasculares y la dermis que aparecen como zonas radiolúcidas; así como la extensión de las distintas cavidades y zona trabecular del cuerno (figura 9).

En las imágenes de resonancia magnética hemos encontrado diferencias de estructura muy evidentes entre individuos, tanto en la extensión de la cavidad o zona trabecular, como en los patrones de los canales; pero no se han descubierto evidencias de alteraciones patológicas.

Una vez procesadas las imágenes, los datos biométricos obtenidos fueron sometidos a un ANOVA de una vía, considerando los dos grupos mencionados anteriormente (tabla 3). 


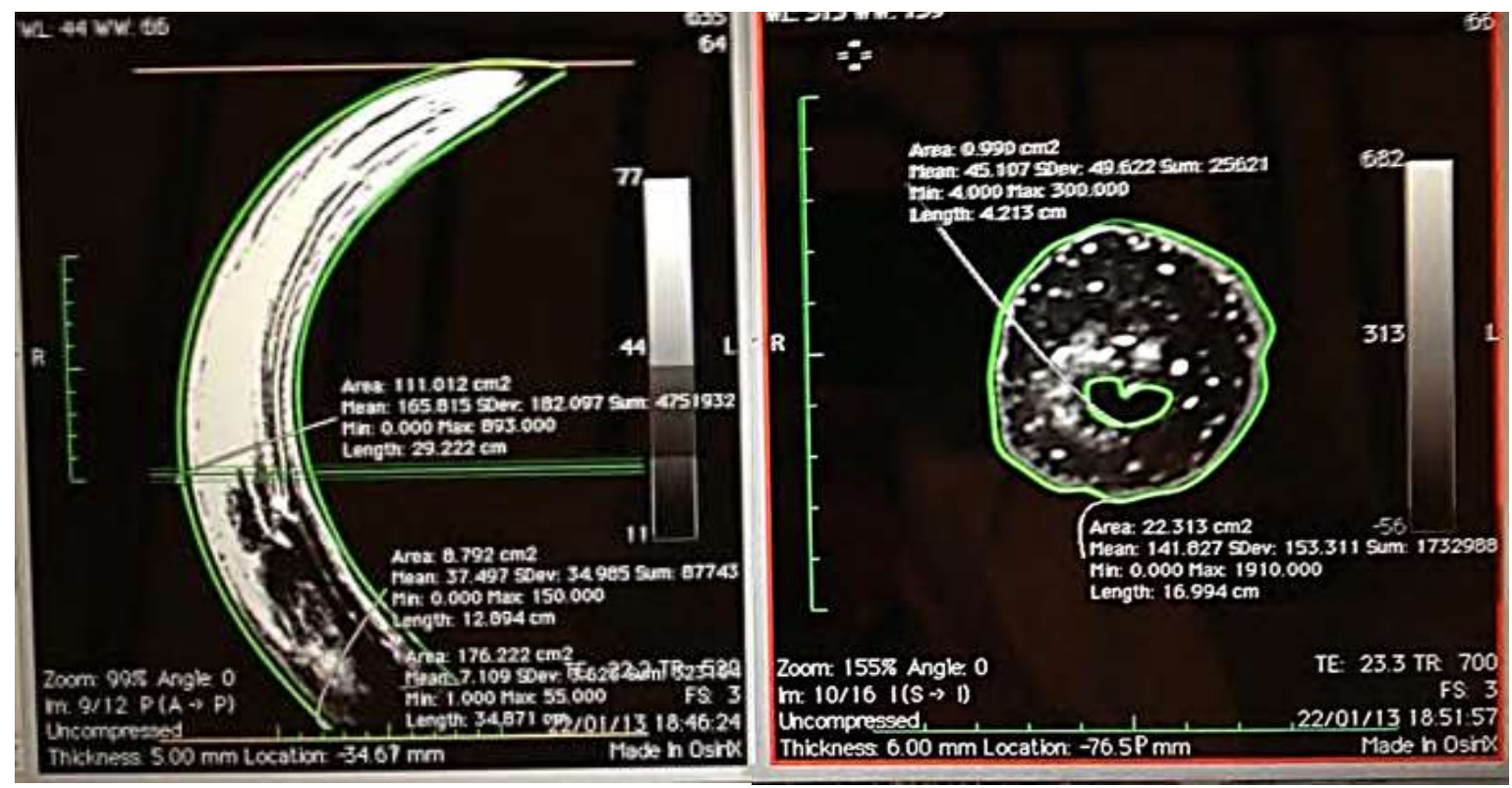

Figura 9. Resonancia magnética de cuerno; corte coronal y axial con una zona trabecular de $12.9 \mathrm{~cm}$ de longitud externa

Tabla 3. ANOVA de los valores biométricos obtenidos mediante resonancia magnética

\begin{tabular}{lccccccccc} 
& Media 0 & Media 1 & $\begin{array}{c}\text { Des. } \\
\text { Est. 0 }\end{array}$ & $\begin{array}{c}\text { Des. } \\
\text { Est.1 }\end{array}$ & df & df & F & p \\
& & & & & & & & \\
\hline Long Total Externa & 43.462 & 43.923 & 3.020 & 3.343 & 1 & 27 & 0.149 & 0.703 \\
Long Macizo & 23.878 & 28.712 & 4.353 & 7.923 & 1 & 27 & 3.872 & 0.059 \\
Long Trabecular & 19.584 & 15.211 & 4.401 & 8.090 & 1 & 27 & 3.050 & 0.092 \\
Long Total Interna & 33.564 & 34.673 & 2.720 & 3.151 & 1 & 27 & 1.000 & 0.326 \\
Área Superficie Total & 23.364 & 27.030 & 3.933 & 11.924 & 1 & 27 & 1.122 & 0.299 \\
Área Maciza & 21.488 & 25.304 & 3.738 & 10.448 & 1 & 27 & 1.562 & 0.222 \\
Área Trabecular & 1.304 & 1.555 & 0.649 & 1.827 & 1 & 27 & 0.223 & 0.641 \\
Perímetro Trabecular & 4.804 & 4.515 & 1.193 & 2.216 & 1 & 27 & 0.179 & 0.676 \\
Perímetro Total & 17.317 & 18.507 & 1.525 & 3.767 & 1 & 27 & 1.139 & 0.295 \\
\hline
\end{tabular}

Pudimos comprobar que no existían diferencias significativas en ninguna de las variables consideradas, aunque la longitud de la zona maciza que no tiene estructura trabecular se encontraba próxima al nivel de significación; siendo superior la parte sin zona trabecular en los cuernos enfundados.

Se realizó una Matriz de correlación lineal entre los parámetros biométricos obtenidos mediante resonancia magnética y la edad de los animales, sin que mostrara ninguna correlación significativa, encontrándose dentro de la misma ganadería animales con longitudes de la zona trabecular muy variables; lo cual no nos ha permitido desarrollar una explicación para estas variaciones (tabla 4). 
Tabla 4. Matriz de correlación lineal entre valores biométricos obtenidos mediante resonancia magnética y la edad

\begin{tabular}{lc}
\cline { 2 - 2 } & Edad \\
\hline Long Total Externa & -0.07 \\
Long Macizo & 0.22 \\
Long Trabecular & -0.24 \\
Long Total Interna & 0.18 \\
Área Superficie Total & 0.82 \\
Área Maciza & 0.47 \\
Área Trabecular & 0.80 \\
Perímetro Trabecular & 0.85 \\
Perímetro Total & 0.83 \\
\hline
\end{tabular}

\section{CONCLUSIONES}

El estudio radiológico ha permitido detectar alteraciones estructurales leves en los cuernos valorados; por ello parece un buen método para la valoración de la integridad de la estructura interna del cuerno. La cornamenta de los animales no enfundados presentó mayores valores de área ósea, contenido mineral y densidad mineral a nivel de la zona ósea distal. No se observaron diferencias en el estudio realizado mediante resonancia magnética nuclear entre los cuernos enfundados y no enfundados; encontrándose grandes variaciones individuales que no se pudieron correlacionar con ninguna de las variables estudiadas.

\section{AGRADECIMIENTOS}

Este trabajo ha sido parcialmente financiado por la Asociación de Veterinarios Taurinos de España (AVET). Agradecemos el uso del equipo de resonancia magnética a la Fundación Sanitaria de León.

\section{LITERATURA CITADA}

ALONSO ME, Lomillos JM, González JR. 2016. La cornamenta del toro de lidia análisis de su integridad y efecto del enfundado. León: EOLAS Ediciones. España. ISBN: 978-84-16613-47-2.

APARICIO-SÁNCHEZ G. 1960. Exterior de los grandes animales domésticos. Imprenta Moderna. Córdoba. España.

APARICIO JB, Peña F, Barona LF. 2000. Estudio de las encornaduras del Toro de Lidia. Ed. Junta de Andalucía. Córdoba. España. ISBN: 846063132X.

BARGA R. 1972. El "afeitado": un fraude de la fiesta brava. Editora Nacional. Madrid. España.

BOBED L. 1982. Estudios sobre crecimiento del asta en reses de lidia. Tesina de Licenciatura. Universidad de Zaragoza. Zaragoza. España. 
CABANAS JM, Gualda MJ, Rosa M y Del Pino J. 1994. Estudio de la composición mineral de las astas del Toro de lidia. I Congreso Mundial de Veterinaria Taurina. Zaragoza. España.

CALVO LA. 2005. Escuela gráfica de toros. Valladolid: Colegio Oficial de Veterinarios de Valladolid. España. ISBN: 9788460972730.

COSSíO JM. 1967. Los toros: tratado técnico e histórico. Espasa Calpe. Madrid. España. ISBN: 9788423993734.

EZPELETA E. 1999. Biometría de los cuernos de las reses de lidia. IV Symposium Nacional del Toro de lidia. Zafra. España. Pp. 227-325.

FERNÁNDEZ J. 2009. Atlas gráfico de pelajes y encornaduras. Curso básico de Espectáculos Taurinos. Madrid: Ed. Colegio de Veterinarios de Madrid. España.

FUENTE D, Ballesteros JP, Guerra J, Morales J, Carpintero M, Durán M, Fernández C, Fernández J, Flores B, Hebrero C, Moreno F, Urquía JJ. 1999. Estudio estadístico de los cuernos lidiados durante las temporadas 1998 y 1999 en la plaza de toros de las Ventas en función del encaste de procedencia e importancia de la lupa binocular en el análisis de los mismos. IV Simposio Nacional del Toro de lidia. Zafra. España. Pp. 249-253.

GÓMEZ FJ, Fernández FJ, Pizarro M, Carpintero CM y Durán JM. 2009. Fractura de pitón durante la lidia, hipotéticamente asociado al enfundado de los cuernos. IX Simposio Nacional del Toro de Lidia. Zafra. España. Pp. 285-288.

HORCAJADA FJ, Fernández C, Ortuño S, Pizarro M. 2009. Diferencias físicas de comportamiento entre pitones enfundados y sin enfundar con vendas de resina de fibra de vidrio: prueba de compresión simple. IX Simposio Nacional del Toro de Lidia. Zafra. España. Pp. 237-242.

LOMILLOS JM, Alonso ME, Gaudioso V. 2013. Análisis de la evolución del manejo en las explotaciones de toro de lidia. Desafíos del sector. Revista ITEA. 109 (1):4968.

https://www.aida-itea.org/index.php/revista-itea/contenidos?idArt=220\&lang=esp

LLORENTE J. 1980. Aportaciones técnicas a la actuación pericial veterinaria en las corridas de toros. Estudio biométrico del asta y constantes bioquímicas hemáticas. Tesis Doctoral. Universidad de Zaragoza. Zaragoza. España.

MARTÍN R. 1984. Estudio anatómico y biométrico de la cornamenta del toro de lidia. Medicina Veterinaria. 1(11):545-553. 
MAUBON P. 1956. La corne du taureau de combat. Tesis Doctoral. Facultad de Veterinaria de la Universidad de Alfort. Alfort. Francia.

PIZARRO M, Carceller H, Alonso R, Horcajada J, Hebrero C. 2008a. Utilización de fundas en cuernos I: Colocación e incidencia en el reconocimiento y comportamiento. VI Congreso Mundial Taurino de Veterinaria. Murcia. España. Pp. 175- 178.

PIZARRO M, Alonso R, Ortuño S y Fernández C. 2008b. Utilización de fundas en cuernos II: Posible modificación de la estructura y consistencia. VI Congreso Mundial Taurino de Veterinaria. Murcia. España. Pp. 179-182.

PIZARRO M, Horcajada FJ, Fernández C, Ortuño S. 2009. Diferencias estructurales entre pitones enfundados y sin enfundar con vendas de resina de fibra de vidrio. IX Simposio Nacional del Toro de Lidia. Zafra. España. Pp. 285-288.

REAL DECRETO 176/1992. Reglamento de espectáculos taurinos. BOE. 56:75707583. España. https://www.boe.es/eli/es/rd/1992/02/28/176

SALAMANCA P. 2009. Análisis de la dureza en cuernos de machos de Lidia de tres y cuatro años de edad comparando el factor encaste. IX Simposio Nacional del Toro de Lidia. Zafra. España. Pp. 263-274.

SAÑUDO C. 2009. Valoración morfológica de los animales domésticos. Ministerio de Medio Ambiente y Medio Rural y Marino. Madrid. España.

https://www.mapa.gob.es/es/ganaderia/temas/zootecnia/LIBRO\%20valoracion\%20 morfologica\%20SEZ_tcm30-119157.pdf

SOTILLO F, Ramírez AR, Sotillo JL. 1996. Biotipología del Toro de Lidia. En: Producciones equinas y de ganado de Lidia. Cap. XV. Zootecnia, bases de producción animal. Tomo XI. Ed. Mundiprensa. Madrid. España. ISBN: 9788471146380.

TRILLO F. 1961. Estudio métrico del asta del toro de lidia y su aplicación práctica. Archivos de Zootecnia. 39(10):34-67. 